\title{
SUPPLEMENTARY MATERIAL TO REUSE, RECYCLE, REWEIGH: COMBATING INFLUENZA THROUGH EFFICIENT SEQUENTIAL BAYESIAN COMPUTATION FOR MASSIVE DATA
}

\author{
By Jennifer A. Tom, Janet S. Sinsheimer \\ AND Marc A. Suchard
}

Details of Sampling From the Complete Model. We include here details about sampling from the complete model outlined in Section 4.3. To sketch out the other update steps for $(\boldsymbol{\theta}, \boldsymbol{\Sigma}, \boldsymbol{\beta}, \boldsymbol{\Gamma})$ in the Gibbs cycle, we define for convenience $\boldsymbol{\Gamma}^{(h \rightarrow 0)}$ as the $\operatorname{diag}\left(\gamma_{1}, \ldots, \gamma_{J-1}\right)$ where $\gamma_{h}$ for $h \in$ $\{1, \ldots, J-1\}$ is replaced by 0 and likewise for $\boldsymbol{\Gamma}^{(h \rightarrow 1)}$ where $\gamma_{h}$ is replaced by 1 . This definition yields the two density ordinates

$$
\begin{aligned}
& \mathrm{N}^{(h \rightarrow 0)} \propto e^{-\frac{1}{2} \operatorname{tr}\left(\boldsymbol{\Sigma}^{-1} \sum_{j=1}^{J}\left(\boldsymbol{T}_{\cdot j}-\boldsymbol{\theta}-\boldsymbol{Z}_{j} \boldsymbol{\Gamma}^{(h \rightarrow 0)} \boldsymbol{\beta}\right)\left(\boldsymbol{T}_{\cdot j}-\boldsymbol{\theta}-\boldsymbol{Z}_{j} \boldsymbol{\Gamma}^{(h \rightarrow 0)} \boldsymbol{\beta}\right)^{\prime}\right)}, \text { and } \\
& \mathrm{N}^{(h \rightarrow 1)} \propto e^{-\frac{1}{2} \operatorname{tr}\left(\boldsymbol{\Sigma}^{-1} \sum_{j=1}^{J}\left(\boldsymbol{T} \cdot j \cdot \boldsymbol{\theta}-\boldsymbol{Z}_{j} \boldsymbol{\Gamma}^{(h \rightarrow 1)} \boldsymbol{\beta}\right)\left(\boldsymbol{T} \cdot{ }_{\cdot j}-\boldsymbol{\theta}-\boldsymbol{Z}_{j} \boldsymbol{\Gamma}^{(h \rightarrow 1)} \boldsymbol{\beta}\right)^{\prime}\right)} .
\end{aligned}
$$


The remaining full conditional distributions are:

$$
\begin{aligned}
& P(\boldsymbol{\theta} \mid \boldsymbol{\Sigma}, \boldsymbol{\Gamma}, \boldsymbol{\beta}, \boldsymbol{T}, \boldsymbol{D}) \\
& \left.\sim \mathrm{N}\left(\left(J \boldsymbol{\Sigma}^{-1}+\tau_{\boldsymbol{\theta}} \mathbf{I}_{I}\right)^{-1}\left(\boldsymbol{\Sigma}^{-1} \sum_{j=1}^{J} \mathbf{X}_{j}+\tau_{\boldsymbol{\theta}} \mu_{\boldsymbol{\theta}} \mathbf{I}_{I}\right),\left(J \boldsymbol{\Sigma}^{-1}+\tau_{\boldsymbol{\theta}} \mathbf{I}_{I}\right)^{-1}\right)\right) \\
& P(\boldsymbol{\beta} \mid \boldsymbol{\theta}, \boldsymbol{\Sigma}, \boldsymbol{\Gamma}, \boldsymbol{T}, \boldsymbol{D}) \\
& \sim \mathrm{N}\left(\left(\sum_{j=1}^{J} \mathbf{L}_{j}+\tau_{\boldsymbol{\beta}} \mathbf{I}_{J}\right)^{-1}\left(\sum_{j=1}^{J} \boldsymbol{\Gamma} \boldsymbol{Z}_{j}^{\prime} \boldsymbol{\Sigma}^{-1}\left(\boldsymbol{T}_{\cdot j}-\boldsymbol{\theta}\right)+\tau_{\boldsymbol{\beta}} \mu_{\boldsymbol{\theta}} \mathbf{I}_{J}\right)\right. \\
& \left.\quad\left(\sum_{j=1}^{J} \mathbf{L}_{j}+\tau_{\boldsymbol{\beta}} \mathbf{I}_{J}\right)^{-1}\right), \\
& P\left(\boldsymbol{\Sigma}^{-1} \mid \boldsymbol{D}, \boldsymbol{\beta}, \boldsymbol{\Gamma}, \boldsymbol{T}, \boldsymbol{\theta}\right) \\
& \sim \mathrm{Wishart}(J+\nu, \\
& \left.\quad\left(\mathbf{R}+\sum_{j=1}^{J}\left(\boldsymbol{T} \cdot j-\boldsymbol{\theta}-\boldsymbol{Z}_{j} \boldsymbol{\Gamma} \boldsymbol{\beta}\right)\left(\boldsymbol{T} \cdot_{j}-\boldsymbol{\theta}-\boldsymbol{Z}_{j} \boldsymbol{\Gamma} \boldsymbol{\beta}\right)^{\prime}\right)^{-1}\right) \\
& P\left(\gamma_{h} \mid \boldsymbol{D}, \boldsymbol{\Sigma}, \boldsymbol{T}, \boldsymbol{\beta}, \boldsymbol{\theta}, \gamma_{-h}\right) \sim \operatorname{Bernoulli}\left(\frac{\mathrm{N}^{(h \rightarrow 1)} p_{0}}{\mathrm{~N}^{(h \rightarrow 1)} p_{0}+\mathrm{N}^{(h \rightarrow 0)}\left(1-p_{0}\right)}\right)
\end{aligned}
$$

where $\mathbf{X}_{j}=\boldsymbol{T}_{\cdot j}-\boldsymbol{Z}_{j} \boldsymbol{\Gamma} \boldsymbol{\beta}, \mathbf{L}_{j}=\left(\boldsymbol{Z}_{j} \boldsymbol{\Gamma}\right)^{\prime} \boldsymbol{\Sigma}^{-1} \boldsymbol{Z}_{j} \boldsymbol{\Gamma}$ and $\mathbf{I}$. is the identity matrix whose dimension is given by its index. The total number of parameters we estimate in the complete model are 8 from the fixed segment effects, 11 from the parameters for time, 11 from variable selection and 36 from the variance matrix or 66 parameters total. See Figure S1 for a schematic of sampling.

Sampling from a constrained covariance structure such as CS (as in Section 4.4.1) requires a Metropolis-Hastings step. We propose $\sigma^{2^{*}}$ via a normally distributed random walk reflected at 0 and $\rho^{*}$ via a windowed-uniform random walk reflected if $\operatorname{abs}\left(\rho^{*}\right)>1$. Let $\boldsymbol{\xi}^{*}=\left(\sigma^{2^{*}}, \rho^{*}\right)$ be the proposed values given the current value. The acceptance probability for a proposed 


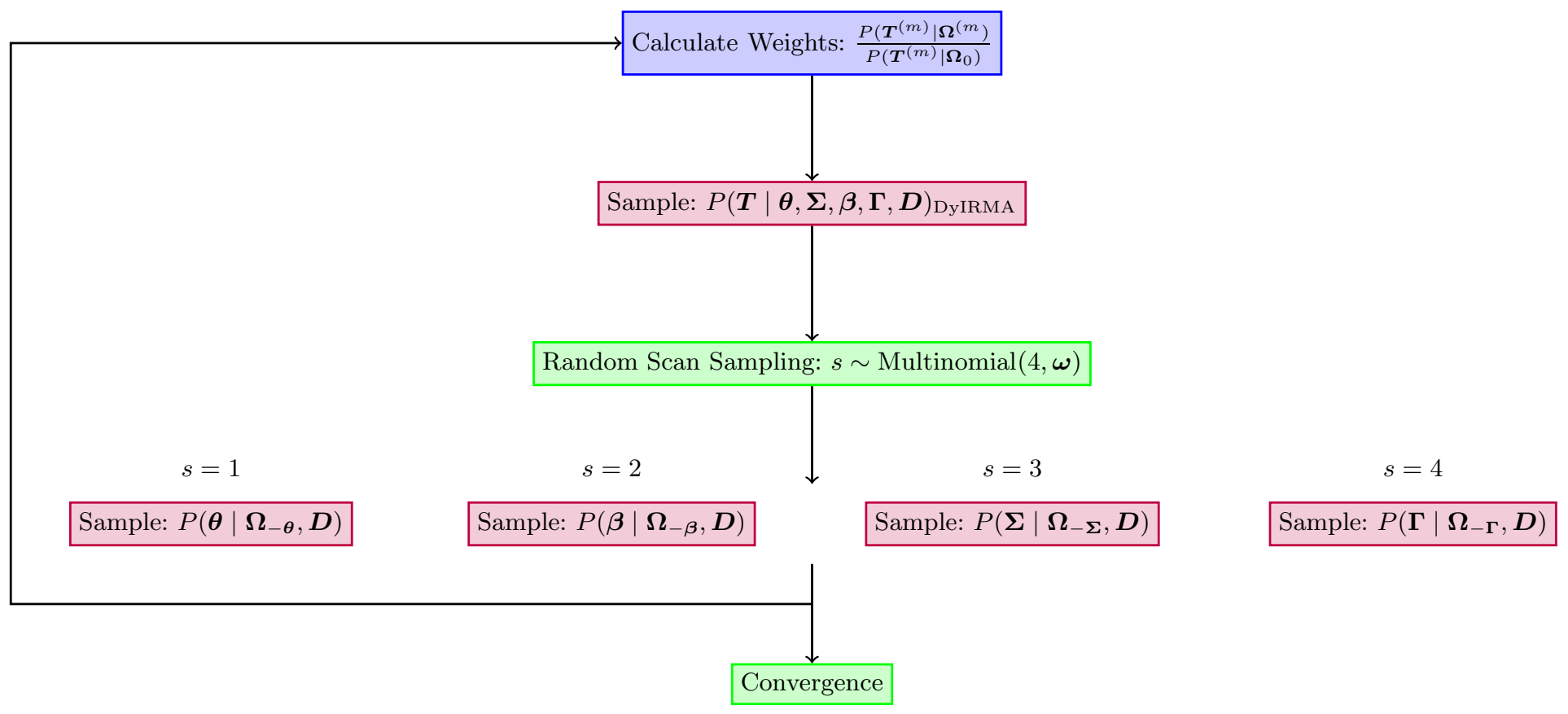

FIG S1. Computational Implementation. Schematic outlining sampling at the $m^{\text {th }}$ step for our proposed model. Sampling continues until convergence. As a reminder, $\boldsymbol{\Omega}=(\boldsymbol{\theta}, \boldsymbol{\Sigma}, \boldsymbol{\beta}, \boldsymbol{\Gamma}, \boldsymbol{T})$ and let $\boldsymbol{\Omega}_{-\boldsymbol{\theta}}=(\boldsymbol{\Sigma}, \boldsymbol{\beta}, \boldsymbol{\Gamma}, \boldsymbol{T})$. Define $\boldsymbol{\Omega}_{-\boldsymbol{\Sigma}}, \boldsymbol{\Omega}_{-\boldsymbol{\beta}}, \boldsymbol{\Omega}_{-\boldsymbol{\Gamma}}$ similarly and the symbol $\boldsymbol{\omega}$ as random scan weights 
4

$\boldsymbol{\Sigma}^{*}=\Psi\left(\boldsymbol{\xi}^{*}\right)$ is $\min \left(1, \alpha_{\boldsymbol{\xi}}\right)$ where we calculate $\alpha_{\boldsymbol{\xi}}$ is as follows:

(S3)

$$
\begin{aligned}
\alpha_{\boldsymbol{\xi}} & =\frac{P\left(\boldsymbol{\xi}^{*} \mid \boldsymbol{\Gamma}, \boldsymbol{\beta}, \boldsymbol{\theta}, \boldsymbol{T}, \boldsymbol{D}\right)}{P(\boldsymbol{\xi} \mid \boldsymbol{\Gamma}, \boldsymbol{\beta}, \boldsymbol{\theta}, \boldsymbol{T}, \boldsymbol{D})} \\
& =\frac{P\left(\boldsymbol{\xi}^{*}\right)\left|\boldsymbol{\Sigma}^{-\frac{J}{2}}\right| e^{-\frac{1}{2} \operatorname{tr} \boldsymbol{\Sigma}^{-1^{*}} \sum_{j=1}^{J}\left(\boldsymbol{T}_{\cdot j}-\boldsymbol{\theta}-\boldsymbol{Z}_{j} \boldsymbol{\Gamma} \boldsymbol{\beta}\right)\left(\boldsymbol{T}_{\cdot j}-\boldsymbol{\theta}-\boldsymbol{Z}_{j} \boldsymbol{\Gamma} \boldsymbol{\beta}\right)^{\prime}}}{P(\boldsymbol{\xi})\left|\boldsymbol{\Sigma}^{-\frac{J}{2}}\right| e^{-\frac{1}{2} \operatorname{tr} \boldsymbol{\Sigma}^{-1} \sum_{j=1}^{J}\left(\boldsymbol{T}_{\cdot j}-\boldsymbol{\theta}-\boldsymbol{Z}_{j} \boldsymbol{\Gamma} \boldsymbol{\beta}\right)\left(\boldsymbol{T}_{\cdot j}-\boldsymbol{\theta}-\boldsymbol{Z}_{j} \boldsymbol{\Gamma} \boldsymbol{\beta}\right)^{\prime}} .} .
\end{aligned}
$$

